\title{
Estructura Factorial de la Versión Breve de Escala Parental Sense of Competence (PSOC) en una Muestra Chilena
}

\author{
Factorial Structure of Short Version of Parental Sense of Competence (PSOC) in a \\ Chilean Sample
}

\author{
Carlos Calderón Carvajal ${ }^{1}$, Siu Lay-Lisboa ${ }^{1}$, Rodrigo Moya-Vergara ${ }^{1}$, Mónica Guzmán-González ${ }^{1}$ \\ y Jimena Silva Segovia ${ }^{1}$
}

\section{Resumen}

El objetivo de este estudio es evaluar la estructura interna de la versión breve del Parental Sense of Competence (PSOC) en una muestra chilena. El análisis de los datos se ha llevado a cabo a través del Análisis Factorial Confirmatorio (AFC) y el Modelo de Ecuaciones Estructurales Exploratoria (ESEM). Adicionalmente hemos estudiado la invarianza de medida entre madres y padres a través del Análisis Factorial Confirmatorio Multi-grupo (AFCM). Los resultados muestran la presencia de dos factores, reportados en estudios previos. Además, los resultados del AFCM soportan existencia de Equivalencia/Invarianza de Medida. Estos resultados son una importante evidencia que apoya la validez de las puntuaciones de la versión reducida del PSOC.

Palabras clave: competencias parentales, estructura factorial, PSOC, invarianza de medida, validez

\begin{abstract}
The aim of this study is to evaluate the internal structure of the short version of the parental sense of competence (PSOC) in a Chilean sample. Data analysis has been carried out through Factorial Confirmatory Analysis (AFC) and the Exploratory Structural Equations Model (ESEM). Additionally we have assessed the measurement invariance among mothers and fathers through the Multi-group Confirmatory Factor Analysis (AFCM). The results show the presence of two factors, which are reported in previous studies. In addition, the results of the AFCM support the existence of Equivalence/Measurement Invariance. These results are important evidence supporting the scale validity of the reduced version of the PSOC.
\end{abstract}

Keywords: parental competences, factorial structure, PSOC, measurement invariance, validity

Estudio financiado por el Proyecto FondecytRegular 1150016 (El lugar del padre en la cultura minera. Estudio mixto sobre experiencias de paternidad, pareja y sexualidad en trabajadores mineros y no mineros de Antofagasta)

${ }^{1}$ Universidad Católica del Norte Académico. Escuela de Psicología. Avenida Angamos 0610. Antofagasta, Chile. Tel.: +56-55-2355822. Correspondencia: ccalderon@ucn.cl 
Introducción

Desde una perspectiva evolutivo-educativa, los padres y madres tienen como tarea construir un escenario adecuado para el desarrollo de sus hijos/as y apoyarlos en su proceso de aprendizaje (Máiquez, Rodrigo, Martín, \& Vermaes, 2000; Rodrigo \& Palacios, 1998). Es así como el ejercicio de la parentalidad requiere de habilidades y competencias adecuadas para manejar y satisfacer las necesidades físicas, cognitivas, emocionales y sociales que presentan los niños/as a través de su ciclo vital (Suwansujarid, Vatanasomboon, Gaylord, \& Lapvongwatana, 2013).

En este sentido, las creencias que pueden presentar los padres y madres con respecto a su eficacia en el manejo de su propio funcionamiento como tales y su capacidad para ejercer control sobre las demandas ambientales y problemas que puedan surgir en sus hijos/as a nivel social, emocional y conductual, representan un factor importante tanto para el bienestar de los padres y madres como el ajuste de sus hijos/as (Jones \& Prinz, 2005; Márk-Ribiczey, Miklósi \& Szabó, 2016).

Es así como Gilmorey Cuskelly (2009) plantearon que la eficacia parental está fuertemente relacionada con las competencias de padres y madres, y los resultados del desarrollo de sus hijo/as (Jones \& Prinz, 2005). Es decir, mientras más seguros se sientan los padres respecto de sus capacidades como tales, utilizarán estrategias de crianza más eficaces, promoviendo resultados positivos en el desarrollo de sus hijos/as, reduciendo al mínimo los riesgos. Estos resultados a su vez, generan sentimientos de competencias como padres (Gilmore \& Cuskelly, 2009). Por el contrario, padres que presentan niveles más bajo de percepción con respecto a sus competencias parentales no solo presentarían habilidades parentales inadecuadas sino que también tenderían a evitar interacciones con sus hijos/as (Ohan, Leung, \& Johnston, 2000).

Para mejorar las conductas de los roles parentales positivos en las intervenciones con los padres, Suwansujarid et al., (2013) plantearon que es necesario contar con instrumentos válidos y confiables que permitan la evaluación de sus competencias parentales percibidas.Uno de los instrumentos más utilizados para evaluar el sentido de las competencias parentales es el Parental Sense of Competence (PSOC) (e.g. Bui, Zakarian, Laifer, Sager, Chen, Cohen, \& Ohye, 2017; Jones \& Prinz, 2005; Márk-Ribiczey et. al., 2016; Nunes, Jiménez, Menéndez, Ayala-Nunes, \& Hidalgo, 2014; Richmond, 2012).

El PSOC fue desarrollado por GibaudWallstonyWandersman (1978). Esta escala consiste en una medida auto-aplicada compuesta por 17 ítems. No obstante, Johnston y Mash (1989) modificaron y difundieron ampliamente este instrumento proponiendo una versión de 16 ítems. En ambas propuestas es posible observar una estructura de dos factores: Satisfacción y Eficacia Parental. Esta estructura ha sido apoyada por varios trabajos (Karp, Lutenbacher, \& Wallston, 2015; Ngai, Wai-Chi Chan \& Holroyd, 2007; Ohan et al., 2000). Sin embargo, existe evidencia de estructuras alternativas. Por ejemplo, existen trabajos que proponen la presencia de un tercer factor: la Controlabilidad (Gilmore \& Cuskelly, 2009; Menéndez, Jiménez, \& Hidalgo, 2011; Nunes et al., 2014, Rogers \& Mattews, 2004;).

Este instrumento ha sido adaptado en varios países tales como China (Ngai et al., 2007), Portugal (Nunes et al., 2014), Australia (Rogers \& Matthews, 2004), Tailandia (Suwansujarid et al., 2013), Alemania (Miller, 2001), Canadá (Ohan et al., 2000); Brasil (Da Silva \& Aiello, 2009), Hungría (Márk-Ribiczey et. al., 2016) y España (Menéndez et al., 2011).

En este último país, Menéndez et al. (2011) han propuesto una versión breve del instrumento, compuesto por 10 ítems presentados en escala likert de 6 opciones de respuesta $(1=$ no, totalmente en desacuerdo a $6=1$ totalmente de acuerdo). A diferencia de los estudios previos, los autores proponen una estructura de dos factores compuestos por la dimensión original de Eficacia, y un segundo factor que no está presente en otros trabajos y que han denominado Controlabilidad. La Eficacia percibida se refiere a las expectativas que presentan los padres y madres de sentirse capaces para actuar de forma competente y eficaz con sus hijos/as (Johnson \& Mash, 1989). Por su parte, la Controlabilidad alude al grado en el cual los padres se sienten responsables ante las tareas educativas de sus hijos/as (Máiquez et al., 2000; 
Rodrigo, Máiquez, Martín, \& Byrne, 2008).

En esta versión breve de la escala PSOC, Menéndez et al., (2011) proponen prescindir de la sub escala de Satisfacción, puesto que presentaba problemas desde el punto de vista conceptual y psicométrico en cuanto a la pertinencia de algunos ítems. En este sentido, señalan que para evaluar las competencias parentales es más apropiado presentar los ítems en positivo, ya que sentirse satisfecho como progenitor no es lo mismo que no sentirse insatisfecho en ese rol.

El presente estudio es planteado como una continuación del trabajo de Menendez et al., (2011) debido a dos razones. En primer lugar, busca recabar mayor evidencia empírica que soporte la estructura propuesta por Menendez et al., (2011), y en segundo, intenta superar las limitaciones técnicas de este trabajo, fundamentalmente las referidas a las actuales recomendaciones para llevar a cabo el análisis en el contexto del estudio de la validez (e.g. Borsboon, 2006; Gaskin \& Happel, 2014; Izquierdo, Olea, \& Abad, 2014; Laher, 2010; Lloret-Segura, Ferreres-Traver, Hernández-Baeza, \& Tomas-Marco, 2014)

En función de lo planteado, el objetivo del presente estudio es analizar las propiedades psicométricas del Parental Sense of Competence (PSOC) versión breve desarrollada por Menéndez et al., (2011), en el contexto chileno. En concreto, el propósito es analizar la estructura interna de la escala y evaluar la Equivalencia/Invarianza de Medida a través del Análisis Factorial Confirmatorio Multi-grupo (AFCM) entre padres y madres. Nos hemos planteado esta comparación debido a laexistencia de evidencia previa acerca de las diferencias en la construcción social de la maternidad y paternidad (e.g. Martín, Cabrera, León, \& Rodrigo, 2013; Urzúa, Godoy, \& Ocayo, 2011; Woollett \& Parr, 1997).

\section{Método}

\section{Participantes}

Participaron en el estudio 562 personas,todas padres y madres de la ciudad de Antofagasta, Chile. La edad media fue de 42.67 años con una desviación típica de 9.94. De ellas, el 27.2\% fueron hombres y $72.6 \%$ fueron mujeres. En cuanto al estado civil, $41.8 \%$ eran solteros/as,
$48.5 \%$ eran casados/as, $8.6 \%$ eran separados/as o divorciados/as y $1.1 \%$ eran viudos/as. En cuanto al nivel educativo, la mayor proporción de participantes corresponden a las categorías nivel de enseñanza secundaria completa $24.3 \%$, formación técnica superior completa $(21.5 \%)$ y estudios universitarios completos (20.3\%).

\section{Instrumento}

El instrumento estuvo compuesto por varias secciones. La primera de ellas estuvo conformada por una serie de preguntas que tenían como propósito indagar características sociodemográficas (sexo, edad, nivel educativo, etc.).

La segunda sección incluyó la escala Parental Sence of Compotence (PSOC) de Johnson yMash (1989) en la versión de Menendez, Jimeneze Hidalgo (2011). Al igual que la versión Española, los 10 ítems de la escala fueron presentados en escala likert de 6 puntos ( $1=$ totalmente en desacuerdo al $6=$ totalmente de acuerdo). Los autores de la versión española han mostrado una fiabilidad adecuada $(\alpha=.76)$. A diferencia del estudio original, Menendez et al., (2011) han propuesto una nueva estructura factorial compuesta dos factores, un factor de "eficacia"reportado en el estudio original, y un nuevo factor denominado "controlabilidad", el cual ha sido reportado en otros trabajos (Gilmore \& Cuskelly, 2009; Menéndez et al.,, 2011; Nunes et al., 2014; Rogers \& Mattews, 2004). Un comité de jueces expertos no recomendó necesaria la modificación y/o adaptación de los enunciados.

En las siquientes secciones se presentaron una serie de escalas que tenían como propósito evaluar algunas variables asociadas a la relación de pareja, que no formaron parte de este subestudio

\section{Propuesta de análisis}

Ya que el propósito del estudio fue evaluar evidencias referidas a la estructura interna de la Escala de Competencias Parentales PSOC (Johnson \& Mash, 1989), los análisis realizados estuvieron enfocados en el análisis factorial de los datos. La propuesta contempló cuatro etapas. La primera de ellas tuvo como objetivo determinar el número de factores a retener. Para este propósito se utilizaron dos técnicas; el Análisis Paralelo (AP) de Horn (1965) y la comparación del ajuste 
de modelos anidados. Estos opciones han sido seleccionadas en base a las recomendaciones realizadas en una serie de trabajos (e.g. Gaskin \& Happel, 2014; Izquierdo, Olea, \& Abad, 2014; Lloret-Segura, Ferreres-Traver, Hernández-Baeza, \& Tomas-Marco, 2014; Laher, 2010), ya que sistemáticamente han mostrado ser los métodos que tienen mejores resultados. Estas técnicas se recomiendan por sobre otras de amplio uso hasta hoy (Conway \& Huffcutt, 2003; Fabrigar, Wegener, MacCallum, \& Strahan, 1999; Henson \& Roberts, 2006; Park, Dailey, \& Lemus, 2002; Pérez \& Medrano, 2010), pero que poseen pobres resultados. Es el caso de la regla K-1 (autovalores mayores que 1), que sobreestima el número de factores (Costello \& Osbome, 2005) y que depende del número de ítems (Ferrando \& Anguiano-Carrasco, 2010; Lorenzo-Seva, Timmerman, \& Kiers, 2011) o el gráfico de sedimentación o Scree test que depende del criterio subjetivo del investigador.

La segunda etapa considera la estimación del modelo a través del Modelo de Ecuaciones Estructurales Exploratoria (ESEM). Esta técnica corresponde a un hibrido entre el Análisis Factorial Exploratorio (AFE) y el Confirmatorio (AFC), el cual posee las ventajas y supera las limitaciones de ambos. Por un lado proporciona los índices de bondad de ajuste y significación de los parámetros que proporciona el AFC y que no están disponibles en AFE, y por otro, no es tan restrictivo como el AFC en el sentido que estima las saturaciones factoriales de los ítems en los factores que supuestamente no miden (cargas cruzadas). Esto permite no acumular error de especificación de las saturaciones fijadas a cero en un AFC convencional. El método de estimación seleccionado fue Máxima Verosimilitud (ML) aplicado sobre la matriz de covarianzas. Ello debido a que los ítems han sido medidos con más de 5 alternativas de respuesta y ninguno de ellos presenta estadísticos de asimetría y curtosis superiores a 1 en valor absoluto (Lloret-Segura et al, 2014).

En tercer lugar, hemos ajustado y comparado una serie de modelos sugeridos en la etapa anterior a través del AFC. Ello permitirá la acumulación de evidencia empírica que soporte la estructura factorial obtenida en la etapa previa. Por último, para estudiar la
Equivalencia/Invarianza de Medida (E/IM) (Horn \& McArdle, 1992), hemos realizado un Análisis de Invarianza Factorial con el propósito de evaluar la estabilidad de la estructura factorial (Labouvie \& Ruetsch, 1995; Little, 1997), y consecuentemente, la equivalencia de las puntuaciones entre distintos grupos (Drasgow, 1987; Horn \& McArdle, 1992). Como agrupación hemos utilizado la variable sexo, por ser una variable en la que se ha estudiado la invarianza en estudios previos (Johnson \& Mash, 1989; Gilmore $\&$ Cuskelly, 2008).

\section{Resultados}

Las medidas de adecuación muestral muestran un ajuste adecuado de los datos al modelo factorial. La medida KMO alcanza un valor por sobre el criterio recomendable $(\mathrm{KMO}=.825)$. Adicionalmente, la prueba de esfericidad de Bartlett lleva al rechazo de la hipótesis que la matriz empírica es igual auna matriz de identidad $\left(\chi^{2}=1482.877 ; p<.05\right)$.

Como hemos mencionado anteriormente, en primer lugar realizado dos tipos de análisis para determinar el número adecuado de factores a retener. El primero de ellos corresponde al AP de Horn (1965). Este análisis compara los autovalores de los factores empíricos con la media y el percentil 95 de autovalores obtenidos de una serie de matrices generadas de manera aleatoria (500 en nuestro caso) y de las cuales sus ítems no se encuentran relacionados. El principio de la técnica es retener el número de factores que obtienen autovalores superiores a los autovalores de los factores aleatorios. El resto de los factores son considerados residuales. Para este estudio utilizamos el AP con la optimización propuesta por Timmermany Lorenzo-Seva (2011), Minimum Rank Factor. Los resultados del AP lo podemos ver graficados en la Figura 1.

Como es posible observar en la gráfica, los dos primeros factores obtienen porcentajes de varianza $(42.3 \%$ y $23.3 \%$ respectivamente) muy por sobre la media $(17.8 \%)$ y el percentil 95 (20.7\%) del segundo factor aleatorio. A partir del 3 factor en adelante, todos los porcentajes de varianza obtenidos se encuentran por debajo de la media y percentil 95 de los factores aleatorios. 


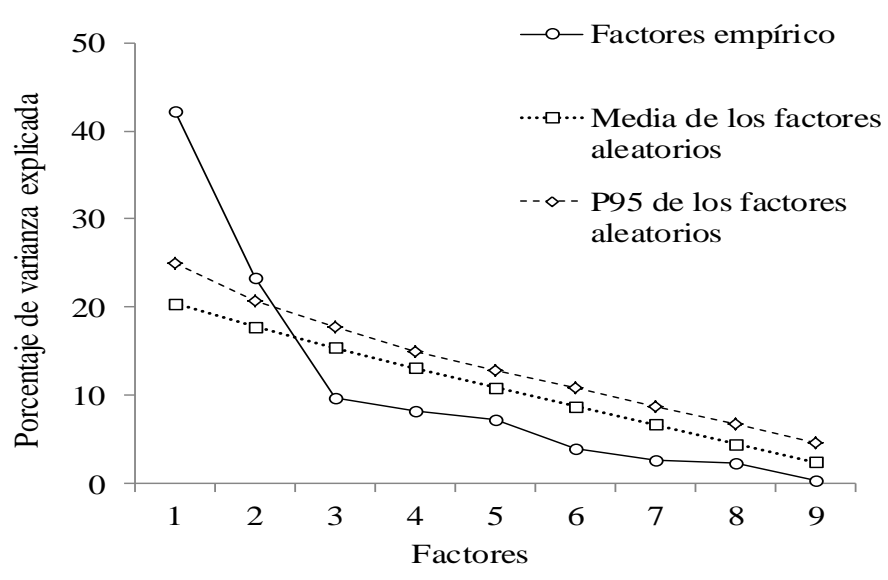

Figura 1. Análisis Paralelo (AP) basado en el método Minimum Rank Factor method de Timmerman \& Lorenzo-Seva (2011)

Tabla 1. Comparación de los estadísticos de bondad de ajuste para los modelos anidados

\begin{tabular}{lccccccc}
\hline Modelos & $\chi 2$ & $\mathrm{gl}$ & $p$-value & $\Delta \chi 2$ & $\Delta \mathrm{gl}$ & $\Delta p$-valor & ${\text { RMSEA }\left(\mathrm{CI}_{90}\right)}^{2}$ \\
\hline 1 factor & 320.603 & 35 & .000 & & & & $.138(.125-.152)$ \\
2 factores & 54.105 & 26 & .001 & 266.498 & 9 & $<.001$. & $.050(.032-.069)$ \\
3 factores & 14.783 & 18 & .677 & 39.323 & 8 & $<.001$ & $.000(.000-.035)$ \\
\hline
\end{tabular}

Tabla 2. Matriz factorial rotada de análisis de Ecuaciones Estructurales Exploratorias (ESEM) Método de estimación de Máxima Verosimilitud con rotación Target

\begin{tabular}{lcc}
\hline & \multicolumn{2}{c}{ Factores } \\
\cline { 2 - 3 } & \multicolumn{1}{c}{1} & 2 \\
\hline $\begin{array}{l}\text { 1. Aunque es difícil, ya he aprendido a cómo influir en mi/s hijo/as } \\
\text { 2. En las cosas que tienen que ver con mi/s hijo/s, me voy a dormir de la misma } \\
\text { manera como me despierto en la mañana, con la sensación de no haber terminado nada } \\
\text { 3. No sé por qué pero, aunque como padre/madre creo que controlo la situación, a } \\
\text { veces siento como si la situación me controlara a mí }\end{array}$ &. $.068 *$ & .068 \\
$\begin{array}{l}\text { 4. Soy capaz de decirle a un/a padre/madre primerizo/a qué es exactamente lo que } \\
\text { tiene que hacer para ser un/a buen/a padre/madre }\end{array}$ & .029 & $.680^{*}$ \\
$\begin{array}{l}\text { 5. Como padre/madre, a veces siento que no doy abasto } \\
\text { 6. He conseguido ser tan buen/a padre/madre como quería }\end{array}$ & $.226^{*}$ & $.137^{*}$ \\
7. Si hay alguien que sabe lo que le pasa a mi/s hijo/s cuando está raro, ese/a soy yo \\
$\begin{array}{l}\text { 8. Teniendo en cuenta el tiempo que llevo siendo padre/madre, me siento muy } \\
\text { familiarizado/a con el rol } \\
\text { 9. Creo que soy capaz de hacer todas las cosas que hacen falta para ser un/a buen/a } \\
\text { padre/madre } \\
\text { 10. Ser padre/madre me hace sentir tenso/a y ansioso/a }\end{array}$ & .063 & $.620^{*}$ \\
\hline
\end{tabular}

Nota. (*) Los parámetros son estadísticamente significativos a nivel .05. Las saturaciones target se presentan en gris.

Este resultado es clara evidencia de la presencia de dos factores.

Una segunda estrategia de retención de factores fue la comparación en el ajuste de modelos anidados a través del estadístico $\chi^{2}$. Adicionalmente, algunos autores (Browne \& Cudeck, 1993; Fabrigar et al., 1999; Ferrando \& Anguiano-Carrasco, 2010; Lorenzo-Seva et al.,
2011) recomiendan la utilización del estadístico RMSEA (Steiger \& Lind, 1980), de manera complementaria. Hemos ajustado tres modelos de uno, dos y tres factores respectivamente. La Tabla 1 nos muestra la comparación de dos modelos ajustados.

De la Tabla 1 podemos desprender dos resultados importantes. En primer lugar el modelo de dos factores presenta un claro desajuste a los 
Tabla 3. Índices de bondad de ajuste de los modelos anidados contrastados a través del AFC

\begin{tabular}{ccccccccccc}
\hline & $\chi^{2}$ & $\mathrm{gl}$ & $p$ & $\Delta \chi 2$ & $\Delta \mathrm{gl}$ & $\Delta p$ & CFI & TLI & SRMR & RMSEA $\left(\mathrm{CI}_{90}\right)$ \\
\hline M1 & 74.249 & 34 & $<.001$ & & & & .972 & .963 & .042 & $.052(.036-.069)$ \\
M2 & 63.542 & 32 & $<.001$ & 10.707 & 2 & .005 & .978 & .970 & .034 & $.048(.030-.065)$ \\
\hline
\end{tabular}

Nota. M1=Modelo de 2 factores; M2; Modelo de 2 factores con 2 croos-loadings en los ítem 4 y 6.

Método de estimación=ML.

Tabla 4. Índices de bondad de ajuste para el análisis de invarianza factorial

\begin{tabular}{lcccccccccc}
\hline & $\chi^{2}$ & $\mathrm{gl}$ & $p$ & $\Delta \chi 2$ & $\Delta \mathrm{gl}$ & $\Delta \mathrm{p}$ & CFI & TLI & SRMR & RMSEA (CI $\left._{90}\right)$ \\
\hline Configural & 130.218 & 62 & $<.001$ & & & & .957 & .943 & .057 & $.066(.048-.083)$ \\
Métrica & 145.435 & 54 & $<.001$ & 15.218 & 8 & .055 & .952 & .944 & .064 & $.066(.049-.082)$ \\
Escalar & 155.686 & 46 & $<.001$ & 10.251 & 8 & .247 & .951 & .947 & .066 & $.064(.048-.079)$ \\
\hline
\end{tabular}

datos. Segundo, aun cuando el estadístico $\chi^{2}$ nos señala que el modelo de 2 factores no ajusta a los datos, el estadístico RMSEA, nos muestra un ajuste adecuado tanto del modelo de 2 como el de 3 factores. Por un criterio de parsimonia, y considerando que el modelo de dos factores, corresponde a la estructura presentada en el estudio original (Johnson \& Mash, 1989) y sistemáticamente encontrada en estudios posteriores, es que nos hemos decantado por el modelo factorial de 2 factores.

Para evaluar la estructura factorial hemos llevado a cabo un análisis de Ecuaciones Estructurales Exploratorias (ESEM). Para el ajuste del modelo hemos utilizado el método de estimación Máxima Verosimilitud con rotación Target sobre la matriz de covarianzas. La matriz target fue especificada según la estructura propuesta por Menéndez, et al., (2011). En cuanto al ajuste global, el modelo factorial estimado alcanzó un ajuste estadístico excelente según todo los índices de ajuste obtenidos $\left(\chi_{26}^{2}=54.932 ; p\right.$ $=.463 ; \quad$ CFI $=.980 ; \quad$ TLI $=.966 ; \quad$ RMSEA $=.051$; SRMR=.024). La Tabla 2 muestra la matriz factorial rotada.

Como es posible observar, la solución factorial obtenida es coherentecon la reportada por Menéndez, et al., (2011) y similar al estudio original (Johnson \& Mash, 1989). En el factor 1 saturan los ítems 1, 4, 6, 7, 8 y 9 y corresponde la dimensión Eficacia. En el factor 2 saturan los ítems 2, 3, 5 y 10 y corresponde a la dimensión Controlabilidad. Cabe señalar dos resultados destacables. En primer lugar, la solución rotada muestra dos cargas cruzadas en los ítems 4 y 6 , los cuales, aunque significativos corresponden a valores muy bajo (.137 y -.086 respectivamente). $\mathrm{Y}$ en segundo lugar, a diferencia de estudios previos (e.g., Menéndez, et al., 2011) la solución no presenta una correlación significativa entre los dos factores ( $r=.004 ; p=.951)$. Con el propósito de determinar la contribución en el ajuste de las cargas cruzadas hemos ajustados a través de AFC y comparado el ajuste entre los modelos de 2 factores con y sin cargas cruzadas en los ítems 4 y 6. El modelo ha sido estimado con el método de Máxima Verosimilitud. Los resultados se presentan en la Tabla 3.

A pesar que el estadístico $\chi^{2}$ muestra un incremento significativo en el ajuste con el modelo con cargas cruzadas (ítems 4 y 6), el resto de los índices de ajuste no muestran un incremento significativo en el ajuste $(\triangle \mathrm{CFI}<.01$; RMSEA<.01; TLI<.01 y SRMR<.025). Con estos resultados nos hemos decantado por el modelo de 2 factores sin cargas cruzadas por ser el modelo más parco.

Finalmente, para determinar la existencia de Equivalencia/Invarianza de Medida hemos realizado un análisis de invarianza factorial utilizando como variables de agrupación el sexo de los participantes (madres vs. padres). Ya que el propósito de este análisis está enfocado en la búsqueda de evidencia que soporte la equivalencia de las puntuaciones de la escala, no hemos incorporado dentro de los modelos anidados la covarianza entre los factores. Los resultados se presentan en la Tabla 4.

Como es posible observar, la totalidad de los índices de bondad de ajuste muestran que no existe una pérdida significativa en el ajuste tanto en los niveles de invarianza métrica como escalar. Estos resultados son evidencia de que no sólo la 
tasa de cambio de los factores latentes (saturaciones factoriales) es equivalente, sino también la media de los factores posee el mismo significado entre los grupos (interceptos). Por tanto es posible afirmar la existencia de invarianza fuerte del modelo factorial entre hombres y mujeres.

Finalmente, en cuanto a los análisis de fiabilidad, ambas dimensiones obtuvieron coeficientes alfa de Cronbach adecuados considerando el número reducido de ítems (Controlabilidad=.829; Eficacia=.683). La Tabla 5 muestra los resultados de las correlaciones ítemtest del coeficiente alfa de Cronbach si se elimina el elemento. Los resultados se presentar por dimensión debido a la estructura ortogonal mostrada por los factores. Como es posible observar, a excepción del ítem 4 (.242), todos los ítems poseen correlaciones superiores a .25. Ello se puede deber a que, como fue presentado anteriormente, el ítem 4 fue el que presentó mayor carga cruzada en las dos dimensiones. En síntesis, resultados muestra que la escala presenta un nivel de fiabilidad adecuado.

Tabla 5. Correlación ítem test y Alfa de Crombach si se elimina el elemento

\begin{tabular}{lcc}
\hline & $\begin{array}{c}\text { Correlacion } \\
\text { item-test }\end{array}$ & $\begin{array}{c}\text { Alfa si se } \\
\text { elimina }\end{array}$ \\
\hline Factor 1: & & \\
Controlabilidad & & \\
Ítem 1 & .546 & .813 \\
Ítem 4 & .242 & .876 \\
Ítem 6 & .666 & .789 \\
Ítem 7 & .718 & .777 \\
Ítem 8 & .746 & .769 \\
Ítem 9 & .760 & .769 \\
Factor 2: Eficacia & & \\
Ítem 2 & .446 & .630 \\
Ítem 3 & .519 & .581 \\
Ítem 5 & .472 & .613 \\
Ítem 10 & .427 & .643 \\
\hline
\end{tabular}

\section{Discusión}

El objetivo del este estudio fue evaluar la estructura interna del Parental Sense of Competence (PSOC) en una muestra chilena. Es una continuación del trabajo de Menéndez, et al., (2011), y busca recabar mayor evidencia empírica acerca de la validez referida a la su estructura factorial. En concreto, nos hemos propuesto dos objetivos. En primer lugar, evaluar la dimensionalidad de la escala a través de una variedad de técnicas actualmente recomendadas para la evaluación de evidencias de validez referida a la estructura interna, y en segundo lugar, estudiar la generalización de estas propiedades métricas evaluando la Equivalencia/Invarianza de Medida, a través del AFCM entre padres y madres. Esta propiedad es condición necesaria para la validez de las comparaciones entre distintos grupos (Casullo, 1999).

Este trabajo aporta nueva evidencia que apoya los estudios previos. Hemos llevado a cabo los análisis siguiendo las recomendaciones en la literatura para el estudio de la estructura interna en el contexto del análisis de evidencias de validez referidas a la estructura interna.

Los resultados apoyan la estructura interna encontrada en trabajos previos. Estas dos dimensiones corresponden a las dimensiones propuestas por Menéndez, et al., (2011): percepción de Eficacia y Controlabilidad Parental. Adicionalmente, los resultados del análisis de Equivalencia/Invarianza de Medida, llevado a cabo a través del AFCM, muestran la existencia de invarianza métrica entre hombres y mujeres. Este hallazgo asegura la equivalencia tanto de las tasas de cambio (invarianza de las saturaciones factoriales) como de la media de los factores (invarianza de los interceptos). Estos resultados son evidencia que apoya la validez de las comparaciones que se pueden realizar entre padres y madres.

A diferencia de estudios previos, los resultados de esta investigación muestran la ortogonalidad de los factores, lo cual indica que las dos dimensiones presentes en los datos no se encuentran relacionadas. Este hallazgo es novedoso, por lo que se requiere mayor evidencia que intente explicar este fenómeno. Adicionalmente, es recomendable intentar replicar estos resultados con otras condiciones (distintas muestras, otros métodos de estimación, etc.), considerando las posibles limitaciones que pueden traer consigo la elección de la matriz empírica o el método de estimación seleccionado en este estudio. A pesar de ello, creemos que los 
hallazgos encontrados son prometedores y son un aporte que apoya la validez referida a la estructura interna de la Escala de Competencias Parentales (PSOC).

\section{Referencias}

Borsboom, D. (2006). The attack of the psychometricians. Psychometrika, 71(3), 425440. doi:10.1007/s11336-006-1447-6

Browne, M. W., \& Cudeck, R. (1992). Alternative Ways of Assessing Model Fit. Sociological Methods \& Research, 21(2), 230-258. doi:10.1177/0049124192021002005

Bui, E., Zakarian, R. J., Laifer, L. M., Sager, J. C., Chen, Y., Cohen, S., \& Ohye, B. (2016). Psychometric Properties of the Parenting Sense of Competence Scale in TreatmentSeeking Post-9/11 Veterans. Journal of Child and Family Studies, 26(2), 464-470. doi:10.1007/s10826-016-0580-9

Conway, J. M., \& Huffcutt, A. (2003). A review and evaluation of exploratory factor analysis practices in organizational research. Organizational Research Methods, 6(2), 147168.doi.org/10.1177/1094428103251541

Casullo, M. (1999). La evaluación psicológica: Modelos, técnicas ycontexto socio-cultural. Revista Iberoamericana de Evaluación - e Avaliação Psicológica, 7, 97-113.

Costello, A. B., \& Osborne, J. (2005). Best practices in exploratory factor analysis: Four recommendations for getting the most from your analysis. Practical Assessment Research \& Evaluation, $\quad 10(7), \quad 1-9$. doi:10.4135/9781412995627.d8

Da Silva, N. B., \& Aiello, A. R. (2009). Análise descritiva do pai da criança com deficiência mental. Descriptive analysis of the father of a mentally disabled child. Estudos de Psicologia, 26(4), 493-503. doi:10.1590/S0103-166X2009000400010

Drasgow, F. (1987). Study of measurement bias of two standardized psychological test. Journal of Applied Psychology, 72(1), 19-29. doi:org/10.1037//0021-9010.72.1.19

Fabrigar, L. R., Wegener, D. T., MacCallum, R. C., \& Strahan, E. J. (1999). Evaluating the use of exploratory factor analysis in psychological re-search. PsychologicalMethods, 4(3), 272299. doi:org/10.1037//1082-989x.4.3.272

Farkas, C. (2008). Escala de Evaluación Parental (EEP): Desarrollo, propiedades psicométricas y aplicaciones. Universitas Psychologica, 7(2), 457-467.

Ferrando, P. J., \& Anguiano-Carrasco, C. (2010). El análisis factorial como técnica de investigación en psicología. Papeles del Psicológo, 31(1), 18-33.

Fyta, K. (2009). Parenting a child with leukemia: Mothers' and fathers' sense of competence and orientation towards uncertainty. Dissertation Abstracts International. Section A, 70, 796.

Gaskin, C., \& Happell, B. (2014). On exploratory factor analysis: A review of recent evidence, an assessment of current practice, and recommendations for future use. International Journal of Nursing Studies. 51(3). 511-521. doi:10.1016/j.ijnurstu.2013.10.005

Gibaud-Wallston, J., \& Wandersman, L. P. (1978). Development and utility of the parenting sense of competence scale. Toronto, Canada: Paper presented at the American Psychological Association. doi:10.1037/t02444-000

Gilmore, L., \& Cuskelly, M. (2009). Factor structure of the Parenting Sense of Competence Scale using a normative sample. Child: Care, Health And Development, 35(1), 48-55. doi:10.1111/j.1365-2214.2008.00867.x

Horn, J. L. (1965). A rationale and test for the number of factors in a factor analysis. Psychometricka, 30(2), 179-185. doi.org/10.1007/bf02289447

Horn, J. L. y McArdle, J. J. (1992). A practical and theoretical guide to measurement invariance in aging research. Experimental Aging Research, 18(3), 117-144. doi.org/10.1080/03610739208253916

Izquierdo, I., Olea, J., \& Abad, F. J. (2014). Exploratory factor analysis in validation studies: Uses and recommendations. Psicothema, 26(3), 395-400.

Jones, T., \& Prinz, R. J. (2005). Potential roles of parental self-eficacy in parent and child adjustment: A review. Clinical Psychology Review, 25(3), 341-363. doi:10.1016/j.cpr.2004 .12.004. 
Johnston, C., \& Mash, E. J. (1989). A measure of parenting satisfaction and efficacy. Journal of Clinical and Child Psychology, 18(2), 167175. doi:10.1207/s15374424jccp1802_8

Karp, S. M., Lutenbacher, M., \& Wallston, K. A. (2015). Evaluation of the Parenting Sense of Competence Scale in mothers of infants. Journal of Child and Family Studies, 24(11), 3474-3481. doi:10.1007/s10826-015-0149-z

Laher, S. (2010). Using exploratory factor analysis in personality research: Best-practice recommendations. Journal of Industrial $\begin{array}{llll}\text { Psychology, } & 36(1), & 7 & \text { pages. }\end{array}$ doi:10.4102/sajip.v36i1.873

Labouvie, E., \& Ruetsch, C. (1995). Testing for equivalence of measurement scales: Simple structure and metric invariance reconsidered. Multivariate Behavioral Research, 30(1), 6376. doi.org/10.1207/s15327906mbr3001_4

Lloret-Segura, S., Ferreres-Traver, A., HernándezBaeza, A., \& Tomás-Marco, I. (2014). El análisis factorial exploratorio de los ítems: Una guía práctica, revisada y actualizada. Anales de Psicología, 30(3), 1151-1169. doi:10.6018/analesps.30.3.199361

Lorenzo-Seva, U., Timmerman, M. E., \& Kiers, H. A. L. (2011). The Hull method for selecting the number of common factors. Multivariate Behavioral Research, 46(2), 340364. doi.org/10.1080/00273171.2011.564527

Máiquez, M. L., Rodrigo, M. J., Capote, C., \& Vermaes, I. (2000). Aprender en la vida cotidiana.Madrid: Visor.

Márk-Ribiczey, N., Miklósi, M., \& Szabó, M. (2016). Maternal self-efficacy and role satisfaction: The mediating effect of cognitive emotion regulation. Journal of Child and Family Studies, 25(1), 189-197. doi:10.1007/s10826-015-0217-4

Martín, J. Cabrera, E. León, J., \& Rodrigo, M. J. (2013). La Escala de competencia y resiliencia parental para madres y padres en contextos de riesgo psicosocial. Anales de Psicología, 29(3), 886-896. doi:10.6018/analesps.29.3.150981

Menéndez, S. Jiménez, L., \& Hidalgo, V. (2011). Estructura factorial de la escala PSOC (Parental Sense of Competence) en una muestra de madres usuarias de servicios de preservación familiar. Revista Iberoamericana de Diagnóstico y Evaluación - e Avaliação Psicológica, 32(2). 187-204.

Miller, Y. (2001). Erziehung von Kindernim Kindergartenalter. Unpublished doctoral dissertation, Technical University of Braunschweig.

Ngai, F., Wai-Chi Chan, S., \& Holroyd, E. (2007). Translation and validation of a chinese version of the parenting sense of competence scale in chinese mothers. Nursing Research,56(5), 348-354.

Nunes, C., Jiménez, L., Menéndez, S., AyalaNunes, L., \& Hidalgo, V. (2014). Psychometric properties of an adapted version of the parental sense of competence (PSOC) scale for portuguese at-risk parents. Child and Family Social Work, 21(4), 433-441. doi:10.1111/cfs.12159

Park, H. S., Dailey, R., \& Lemus, D. (2002). The use of exploratory factor analysis and principal components analysis in communication research. Human Communication Research, 28(4), 562-577. doi.org/10.1093/hcr/28.4.562

Pérez, E., \& Medrano, L. (2010). Análisis factorial exploratorio: Bases Con-ceptuales y Metodológicas. Revista Argentina de Ciencias del Comportamiento, 2(1), 58- 66.

Ohan, J. L., Leung, D. W., \& Johnston, C. (2000). The Parenting Sense of Competence Scale: Evidence of a stable factor structure and validity. Canadian Journal of Behavioural Science / Revue Canadienne Des Sciences Du Comportement, 32(4), 251-261. doi: $10.1037 / \mathrm{h} 0087122$

Richmond, M. P. (2012). A Participatory Action Research (PAR) study of Parenting Sense of Competence (PSOC) in parents of children with spina bifida (SB). Dissertation Abstracts International, 72, 6397.

Rodrigo, M. J., Máiquez, M. L., Martín, J. C., \& Byrne, S. (2008). Preservación familiar. Un enfoque positivo para la intervención con familias. Madrid: Pirámide.

Rodrigo M. J., \& Palacios, J. (1998). Familia y desarrollo humano. Madrid: Alianza Editorial.

Rogers, H., \& Matthews, J. (2004). The parenting sense of competence scale: Investigation of the factor structure, reliability, and validity for 
an Australian sample. Australian Psychologist, $\quad 39(1), \quad$ 88-96. doi:10.1080/00050060410001660380

Steiger, J. H., \& Lind, J. M. (1980). Statistically based tests for the number of common factors. Paper presented at the annual meeting of the Psychometric Society, Iowa City, IA.

Timmerman, M. E., \& Lorenzo-Seva, U. (2011). Dimensionality assessment of ordered polytomous items with parallel analysis. Psychological Methods, 16(2), 209-220. doi:10.1037/a0023353

Suwansujarid, T., Vatanasomboon, P., Gaylord, N., \& Lapvongwatana, P. (2013). Validation of the parenting sense of competence scale in fathers: Thai version. Southeast Asian Journal of Tropical Medicine and Public Health, 44(5), 916.

Urzúa, A. Godoy, J., \& Ocayo, K. (2011). Competencias parentales percibidas y calidad de vida. Revista Chilena de Pediatría, 82(4), 300-310. doi:10.4067/s0370-41062011000400004

Woollett, A., \& Parr, M. (1997). Psychological tasks for women and men in the postpartum. Journal of Reproductive and Infant Psychology, 15(2), 159-183. doi:10.1080/02646839708404541 